\title{
Polymorphisms in genes involved in innate immunity and susceptibility to benzene-induced hematotoxicity
}

\author{
Min Shen ${ }^{1}$, Luoping Zhang ${ }^{2}$, Kyoung-Mu Lee ${ }^{1,3}$, \\ Roel Vermeulen ${ }^{4}$, H. Dean Hosgood ${ }^{1,6}$, Guilan $\mathrm{Li}^{5}$, \\ Songnian Yin $^{5}$, Nathaniel Rothman ${ }^{1}$, \\ Stephen Chanock ${ }^{1}$, Martyn T. Smith ${ }^{2}$ and Qing Lan ${ }^{1}$ \\ ${ }^{1}$ Division of Cancer Epidemiology and Genetics \\ $\mathrm{NCl}, \mathrm{NIH}, \mathrm{DHHS}$, Bethesda \\ MD 20892, U.S.A. \\ ${ }^{2}$ School of Public Health \\ University of California, Berkeley \\ CA 94720, U.S.A. \\ ${ }^{3}$ Department of Environmental Health \\ Korea National Open University \\ Seoul 110-791, Korea \\ ${ }^{4}$ Institute for Risk Assessment Sciences \\ Utrecht University; Julius Center \\ University Medical Center Utrecht \\ Utrecht, Netherlands \\ ${ }^{5}$ Institute of Occupational Health and Poison Control \\ Chinese Center for Disease Control and Prevention \\ Beijing, China \\ ${ }^{6}$ Corresponding author: Tel, 301-594-4649; \\ Fax, 301-402-1819; E-mail, hosgoodd@ mail.nih.gov \\ DOI 10.3858/emm.2011.43.6.041
}

Accepted 24 January 2011

Available Online 3 May 2011

Abbreviations: $\mathrm{CBC}$, complete blood count; FDR, false discovery rate; GEE, generalized estimating equations; NK, natural killer; SD, standard deviation; SNPs, single nucleotide polymorphisms; WBC, white blood cell

\begin{abstract}
Benzene, a recognized hematotoxicant and carcinogen, can damage the human immune system. We studied the association between single nucleotide polymorphisms (SNPs) in genes involved in innate immunity and benzene hematotoxicity in a cross-sectional study of workers exposed to benzene ( 250 workers and 140 controls). A total of 1,236 tag SNPs in 149 gene regions of six pathways were included in the analysis. Six gene regions were significant for their association with white blood cell (WBC) counts (MBP, VCAM1, ALOX5, MPO, RAC2, and CRP) based on gene-region $(P<0.05)$ and SNP analyses (FDR
\end{abstract}

<0.05). VCAM1 rs3176867, ALOX5 rs7099684, and MPO rs2071409 were the three most significant SNPs. They showed similar effects on WBC subtypes, especially granulocytes, lymphocytes, and monocytes. A 3-SNP block in ALOXE3 (rs7215658, rs9892383, and rs3027208) showed a global association (omnibus $P=$ 0.0008) with WBCs even though the three SNPs were not significant individually. Our study suggests that polymorphisms in innate immunity genes may play a role in benzene-induced hematotoxicity; however, independent replication is necessary.

Keywords: benzene; hematology; immunity, innate; polymorphism, single nucleotide; toxicity

\section{Introduction}

Benzene is a component of cigarette smoke, gasoline, crude oil, and automobile emissions, and an important industrial and environmental pollutant. Several million workers worldwide are exposed to benzene in the petroleum industry, shipping, automobile repair, shoe manufacture, etc (Goldstein, 1988). Epidemiological studies have shown that exposure to benzene results in an increased risk of aplastic anemia, myelodysplastic syndromes, leukemia, and other blood disorders (Goldstein, 1988). Benzene metabolites may induce toxicity and leukemogenicity by oxidative stress, DNA damage, growth factor regulation, cell cycle regulation, and/or programmed cell death (Yoon et al., 2003).

Innate immunity is the first barrier of the human body to clear non-specific antigens and interacts with the adaptive immune system during physiological and chronic inflammation (Kabelitz and Medzhitov, 2007). Benzene has been found to damage both the humoral and cellular components of the immune system; decreased levels of antibodies and leukocytes can result from exposure to benzene in animal and human studies (Agency for Toxic Substances and Disease Registry, 2007).

Genetic polymorphisms in genes involved in human innate immunity may modify the defense against benzene and affect susceptibility to benzeneinduced hematotoxicity. We examined the association between single nucleotide polymorphisms (SNPs) in several innate immunity genes and benzeneinduced hematotoxicity in a cross-sectional study conducted in China. These genes were categorized 
in six pathways: oxidative response; pattern recognition molecules/antimicrobials; integrins/receptors; complements; chemokines; and response genes/ tissue factors.

\section{Results}

Demographic characteristics were similar among controls and among benzene-exposed workers (Lan et al., 2004). The majority of the study subjects were females $(65 \%)$ and relatively young $($ mean age $=30($ standard deviation $(S D)=8)$ ). The benzene-exposed workers had been employed for an average of $6.1 \pm 2.9$ years. The average level of benzene in the air in the month prior to phlebotomy was $5.4(\mathrm{SD}=12.1) \mathrm{ppm}$ in the exposed group. Counts of WBCs and most WBC subtypes as well as platelets were significantly reduced in benzene-exposed workers compared with controls (Lan et al., 2004).

All results of SNP analyses are shown in Supplementary Table 2. Table 1 shows the most significant genes and SNPS (MBP, VCAM1, $A L O X 5, M P O, R A C 2$ and $C R P$ ) whose minor allele frequencies were more than 2 percent and $\mathrm{P}<$ 0.05 in permutation test or FDR $<0.05$. VCAM1 rs3176867, ALOX5 rs7099684, and MPO rs2071409 were three most significant SNPs. These SNPs did not have a substantial influence on WBC counts among unexposed subjects. We further evaluated the influence on several major WBC subtypes and platelets of these 8 SNPs (Supplementary Table 3). They showed similar effects on WBC subtypes, especially granulocytes, lymphocytes, and monocytes.

Haplowalk analyses found a strong global association (omnibus $P=0.0008$ ) between WBC counts and a 3-SNP block in ALOXE3, in which the individual SNPs were not significant (rs7215658, P trend $=0.90 ; \mathrm{rs} 9892383, \mathrm{P}$ trend $=0.17 ; \mathrm{rs} 3027208, \mathrm{P}$ trend $=0.33$ ). Haplotype analysis showed that the strong global association was driven by a haplotype that differs from the common haplotype at all 3 loci (A-C-C (7\%) vs. C-T-T (43\%), $\beta=-0.09$ for WBCs, $P=0.04)$. Haplowalk analyses for other gene regions did not provide additional pronounced loci in addition to those significant SNPS found in individual SNP analyses.

\section{Discussion}

We studied the association between genetic polymorphisms in genes that play a role in innate immunity and benzene-induced hematotoxicity. We found that SNPs in several gene regions involved in innate immunity were associated with WBC counts, suggesting that innate immunity may play a role in benzene-induced hematotoxicity.

We previously reported associations between benzene-induced hematotoxicity and SNPs in VCAM1 (rs1041163, -1591 C > T) and MPO (rs2333227, $-463 \mathrm{G}>$ A) (Lan et al., 2004; 2005). We report here findings for an additional SNP in each of these two genes that were more significant than previously reported SNPs and that had moderately high to low linkage with those SNPs (i.e., VCAM1 rs3176867, IVS4-458 C $>$ T $\left(r^{2}=0.69\right) ; \quad M P O$ rs2071409, IVS11-6 A > C $\left.\left(r^{2}=0.26\right)\right)$. Additional analyses with multiple regression models including both SNPs in VCAM1 or both SNPs in MPO indicated that VCAM1 rs3176867 and MPO rs2071409 remained significant and that the previously reported SNPs became non-significant (data not shown). Our new findings provide additional clues for the location of possible causal loci in these two genes.

Previous reports show that benzene can cause damage to the immune system (Agency for Toxic Substances and Disease Registry, 2007). Reduced WBCs and WBC subtypes has been demonstrated in workers exposed to as low as $<1 \mathrm{ppm}$ benzene (Lan et al., 2004). Such immune damage can be induced by either oral or dermal exposures, and both humoral and cell-mediated immunity are affected. In addition, immunological responses after exposure to benzene were found to be biphasic in some studies: proliferative response at low exposure levels and depressed responses at high levels (Agency for Toxic Substances and Disease Registry, 2007).

Innate immunity is the first barrier to protect the host from invasion of exogenous chemicals or microbes. Although innate immunity consists of numerous pathways, such as Pattern Recognition Molecules \& Antimicrobials, Integrins/Receptors, Oxidative Response, and Complement, the innate immunity response relies on integrins and receptors to recognize and respond to foreign substances. Our findings that genetic variation in integrin genes is associated with benzene-hematotoxicity suggest that alterations in the body's ability to respond to exogenous invasion may lead to higher susceptibility to chemical-induced adverse health effects. In support of these findings, immune responses to simple chemicals, such as general allergic contact hypersensitivity and chemical-induced specific cutaneous immunity in contact dermatitis, have been reported to be mediated through activation of the innate immune system (Zhang and Tinkle, 2000). Given that contact dermatitis in 
Table 1. Association between selected genetic polymorphism and WBC counts in benzene-exposed workers and controls

\begin{tabular}{|c|c|c|c|c|c|c|c|c|c|}
\hline Gene & rs\# & & Controls & $\mathrm{WBC}^{1}$ & $P^{2}$ & Exposed & $\mathrm{WBC}^{1}$ & $P^{3}$ & $P^{4}$ \\
\hline \multicolumn{10}{|c|}{ Pattern recognition molecules \& antimicrobials } \\
\hline \multirow[t]{2}{*}{$M B P$} & rs470261 & AA & 101 & $6,514 \pm 174$ & 1 & 222 & $5,465 \pm 133$ & 1 & 0.04 \\
\hline & IVS4 -898T >C & $A G$ & 2 & $6,500 \pm 183$ & 0.87 & 8 & $6,438 \pm 802$ & 0.001 & \\
\hline \multicolumn{10}{|c|}{ Integrins/Receptors } \\
\hline \multirow[t]{10}{*}{ VCAM1 } & rs1041163 & TT & 73 & $6,512 \pm 154$ & 1 & 163 & $5,667 \pm 130$ & 1 & 0.001 \\
\hline & $-1591 \mathrm{C}>\mathrm{T}$ & CT & 26 & $6,804 \pm 214$ & 0.61 & 62 & $5,127 \pm 134$ & 0.011 & \\
\hline & & $\mathrm{CC}$ & 4 & $4,650 \pm 145$ & 0.02 & 5 & $4,620 \pm 646$ & 0.004 & \\
\hline & & $\mathrm{CC} / \mathrm{CT}$ & 30 & $6,517 \pm 217$ & 0.89 & 67 & $5,090 \pm 131$ & 0.004 & \\
\hline & & Trend & & & 0.38 & & & 0.001 & \\
\hline & rs3176867 & $\mathrm{CC}$ & 79 & $6,558 \pm 163$ & 1 & 151 & $5,703 \pm 132$ & 1 & \\
\hline & IVS4 $-458 \mathrm{C}>\mathrm{T}$ & $\mathrm{CT}$ & 17 & $6,753 \pm 213$ & 0.78 & 74 & $5,082 \pm 117$ & 0.001 & \\
\hline & & $\mathrm{TT}$ & 7 & $5,429 \pm 178$ & 0.18 & 4 & $4,425 \pm 550$ & $<0.0001$ & \\
\hline & & TT/CT & 24 & $6,367 \pm 209$ & 0.62 & 78 & $5,049 \pm 116$ & 0.0001 & \\
\hline & & Trend & & & 0.33 & & & $<0.0001$ & \\
\hline \multirow[t]{10}{*}{ ALOX5 } & rs4948671 & TT & 71 & $6,670 \pm 181$ & 1 & 173 & $5,622 \pm 131$ & 1 & 0.008 \\
\hline & IVS2 + 1927C $>T$ & CT & 31 & $6,181 \pm 156$ & 0.17 & 54 & $5,141 \pm 133$ & 0.003 & \\
\hline & & $\mathrm{CC}$ & 1 & 5,700 & & 3 & $4,833 \pm 145$ & 0.12 & \\
\hline & & $\mathrm{CC} / \mathrm{CT}$ & 32 & $6,166 \pm 154$ & 0.13 & 57 & $5,125 \pm 132$ & 0.002 & \\
\hline & & Trend & & & 0.09 & & & 0.001 & \\
\hline & rs7099684 & TT & 79 & $6,670 \pm 174$ & 1 & 172 & $5,633 \pm 130$ & & \\
\hline & IVS3 + 5771A $>$ T & AT & 23 & $6,013 \pm 169$ & 0.06 & 52 & $5,079 \pm 118$ & 0.001 & \\
\hline & & $\mathrm{AA}$ & 1 & 5,700 & & 3 & $4,433 \pm 155$ & 0.03 & \\
\hline & & AA/AT & 24 & $6,000 \pm 165$ & 0.04 & 55 & $5,044 \pm 119$ & 0.0003 & \\
\hline & & Trend & & & 0.02 & & & 0.0001 & \\
\hline \multicolumn{10}{|c|}{ Oxidative response } \\
\hline \multirow[t]{6}{*}{ MPO } & rs2071409 & TT & 85 & $6,486 \pm 173$ & 1 & 192 & $5,382 \pm 125$ & 1 & 0.13 \\
\hline & IVS11 -6A >C & GT & 18 & $6,644 \pm 179$ & 0.36 & 34 & $5,915 \pm 149$ & 0.01 & \\
\hline & & GG & & & & 4 & $7,550 \pm 142$ & $<0.0001$ & \\
\hline & & GG/GT & & & & 38 & $6,087 \pm 155$ & 0.002 & \\
\hline & & Trend & & & & & & & \\
\hline & 0.0001 & & & & & & & & \\
\hline \multirow[t]{5}{*}{ RAC2 } & rs2239773 & GG & 84 & $6,517 \pm 174$ & 1 & 191 & $5,613 \pm 137$ & 1 & 0.03 \\
\hline & IVS1 -591C > T & $A G$ & 17 & $6,794 \pm 161$ & 0.346 & 39 & $4,941 \pm 888$ & 0.002 & \\
\hline & & AA & 2 & $4,000 \pm 0$ & & & & & \\
\hline & & AA/AG & 19 & $6,500 \pm 176$ & 0.859 & & & & \\
\hline & & Trend & & & 0.666 & & & & \\
\hline \multicolumn{10}{|c|}{ Complement } \\
\hline \multirow[t]{2}{*}{$C R P$} & rs1800947 & CC & 98 & $6,487 \pm 174$ & 1 & 218 & $5,477 \pm 134$ & 1 & 0.04 \\
\hline & $E \times 2+491 G>C$ & CG & 5 & $7,040 \pm 185$ & 0.176 & 12 & $5,900 \pm 111$ & 0.003 & \\
\hline
\end{tabular}

${ }^{1}$ Unadjusted total WBC count (/ul) as mean \pm standard deviation.

${ }^{2} P$ values from GEE models adjusted for age, sex, current smoking, current alcohol drinking, BMI, and recent infections.

${ }^{3} P$ values from GEE models adjusted for age, sex, current smoking, current alcohol drinking, BMI, recent infections, In air benzene exposure, and In air toluene exposure in the month prior to phlebotomy.

${ }^{4} P$ values for permutation test for specific gene regions.

humans exposed to benzene oxidation products has also been described our findings are biologically plausible (Basketter and Liden, 1992).

In contrast to innate immunity to microbes, mechanisms of innate immunity to chemicals remain unclear. Benzene metabolites can sensitize the immune system as haptens to induce allergic response through suppressing NFKB binding activity (Kim et al., 2005). They also can interfere with immune responses by inhibiting cytokine pro- duction (Ibuki and Goto, 2004). Hydroquinone, a reactive metabolite of benzene, was found to reduce macrophage-mediated immune responses (Lee et al., 2007).

At the same time, a number of genes with suggestive findings analyzed in this report, which focuses on a panel of genes that are important for innate immunity, also play a role in other processes that are relevant to benzene hematotoxicity. These include hematopoiesis (VCAM1 and RAC2 (Hall 
and Gibson, 2004; Guo et al., 2008)) and metabolic activation of benzene (MPO (Lan et al., 2004)), implying that some of our findings may reflect processes other than innate immunity.

The moderate sample size of our study may lead to both false positive and false negative findings (Wacholder et al., 2004). We accounted for possible spurious findings due to multiple comparisons by evaluating False Discovery Rates. Further, associations with a particular SNP in this study may be the result of linkage disequilibrium with another functional SNP in the region.

Our study suggests that genetic polymorphisms in one or more genes that play a role in innate immunity may modify the risk of hematotoxicity in benzene-exposed workers. At the same time, however, our findings need to be replicated in other independent, larger studies.

\section{Methods}

\section{Study population and exposure assessment}

Details of this cross-sectional study have been described elsewhere (Lan et al., 2004). Briefly, the study population included 250 workers who were exposed to benzene in two shoe manufacturing factories and 140 unexposed controls from comparable populations who worked in three clothing-manufacturing factories in the same region of China. Data were obtained from 28 benzene-exposed workers in both study years (2000 and 2001) and are treated as independent observations in the study. Controls were frequency-matched by sex and age to exposed workers. Blood samples were collected from all workers. Before phlebotomy, individual exposure to benzene and toluene, as well as other organic solvents, was monitored repeatedly up to 16 months by wearing an organic vapor passive monitor badge $v$. Subjects were administered a questionnaire for information on lifetime occupational history, environmental exposures, medical history and current medications, and past and current tobacco and alcohol use. The complete blood count (CBC) was analyzed using a T540 blood counter, and the major lymphocyte subsets were analyzed by a FACS Calibur flow cytometer (Software: SimulSET v3.1).

\section{Genotyping}

A GoldenGate assay (Illumina, http://www.illumina.com) was developed using SNPs with plausible evidence that the gene product is related to innate immunity. Gene region was defined as a chromosome region with multiple genes within a range of 200,000 bases.

Although 1,466 SNPs were successfully genotyped in this study, 102 were excluded due to poor quality performance (low completion rates, concordances, or HardyWeinberg disequilibrium), and 128 were dropped from data analysis because of low minor allele frequency $(<1 \%)$. A total of 1,236 tag SNPs in 149 gene regions (207 genes) were included in the analysis (Supplementary Table 1).

\section{Statistical analysis}

Total white blood cell (WBC) was used as the main endpoint of this study since altered WBC count is a primary component of benzene poisoning diagnosis in China and has been associated with risk of hematologic malignancies and related disorders among benzene-exposed workers (Rothman et al., 1997). Generalized estimating equations (GEE) were used to model the relationship between SNPs and blood cell counts (on log scale), adjusting for a potential correlation between the repeated measurements from the 28 exposed workers that were studied in both years and treated independently (Zeger and Liang, 1986). Exposed and unexposed workers were analyzed separately. The genotype data were analyzed by comparing the homozygotes and the heterozygotes of the variant with the homozygote of the common allele. An additive model was applied by treating the genotypes as values of 0,1 , and 2 in one model in order to test for a linear trend. The relationship between each tagSNP and WBC count was evaluated adjusting for age (continuous variable), sex, current cigarette smoking status (yes/no), current alcohol consumption (yes/no), recent infections (yes/no), and body mass index (BMI). For analyses restricted to benzene exposed workers, the model was also adjusted for the natural log (In) mean air benzene and In mean air toluene exposure in the month prior to phlebotomy. Gene-benzene interactions were estimated by adding a multiplicative term between SNP (variant carriers vs. wild type carriers) and benzene exposure (yes/no) into a model with all subjects.

We evaluated the robustness of individual SNP analyses using the False Discovery Rate (FDR) (Benjamini and Hochberg, 1995). We evaluated the genetic association at the gene region level using a permutation-based resampling method (1,000 permutations) to assess the true statistical significance of the smallest $P$-trend within each gene region (Chen et al., 2006).

Haplotype analysis was carried out for all gene regions. Haplotype frequencies were estimated using the expectationmaximization algorithm (Excoffier and Slatkin, 1995) and haplotypes with frequencies less than $1 \%$ were collapsed into a single category. All contiguous locus subsets in a gene region were scanned to identify significant subhaplotypes in exposed workers, adjusting for the same covariates in SNP analyses (Chen et al., 2006). For significant sub-haplotypes, the association of individual haplotypes with WBCs was estimated using a GEE model with the most common haplotype as the reference (Chen et al., 2006). A two tagSNP sliding window was also performed to identify regions associated with altered WBC counts (Huang et al., 2007).

SNPs demonstrating significant gene-dosage effects (i.e., trend test) on WBCs among workers exposed to benzene were further explosred for their influence on specific WBC subtypes (granulocytes, lymphocytes, CD4-T cells, CD8-T cells, CD4/CD8 ratio, B Cells, natural killer (NK) cells, and monocytes) and platelets. All analyses were conducted using the Statistical Analysis Software, version 9.13 (SAS Institute Inc, 1996) if not specified otherwise. 


\section{Supplemental data}

Supplemental Data include three tables and can be found with this article online at http://e-emm.or.kr/article/article_files/ SP-43-6-07.pdf.

\section{Acknowledgements}

This project was in part by the $\mathrm{NIH}$ intramural research program, and by NIH grants RO1ES06721, P42ES04705 and P30ES01896 (to MTS).

\section{References}

Agency for Toxic Substances and Disease Registry 2007., Toxicological Profile for Benzene, U.S. DEPARTMENT OF HEALTH AND HUMAN SERVICES, Atlanta, Georgia, CAS No. $71-43-2$

Basketter DA, Liden C. Further investigation of the prohapten concept: reactions to benzene derivatives in man. Contact Dermatitis 1992;27:90-7

Benjamini Y, Hochberg Y. Controlling the False Discovery Rate: a practical and powerful approach to multiple testing. J. of the Royal Stat. Society B 1995;57:289-300

Chen BE, Sakoda LC, Hsing AW, Rosenberg PS. Resampling-based multiple hypothesis testing procedures for genetic case-control association studies. Genet Epidemiol 2006;30:495-507

Excoffier L, Slatkin M. Maximum-likelihood estimation of molecular haplotype frequencies in a diploid population. Mol Biol Evol 1995;12:921-7

Goldstein BD. Benzene toxicity. Occup Med 1988;3: 541-54

Guo F, Cancelas JA, Hildeman D, Williams DA, Zheng Y. Rac GTPase isoforms Rac1 and Rac2 play a redundant and crucial role in T-cell development. Blood 2008;112:1767-75

Hall BM, Gibson LF. Regulation of lymphoid and myeloid leukemic cell survival: role of stromal cell adhesion molecules. Leuk Lymphoma 2004;45:35-48

Huang BE, Amos CI, Lin DY. Detecting haplotype effects in genomewide association studies. Genet Epidemiol 2007;31: 803-12

Ibuki Y, Goto R. Dysregulation of apoptosis by benzene metabolites and their relationships with carcinogenesis. Biochim Biophys Acta 2004;1690:11-21
Kabelitz D, Medzhitov R. Innate immunity--cross-talk with adaptive immunity through pattern recognition receptors and cytokines. Curr Opin Immunol 2007;19:1-3

Kim E, Kang BY, Kim TS. Inhibition of interleukin-12 production in mouse macrophages by hydroquinone, a reactive metabolite of benzene, via suppression of nuclear factor-kappaB binding activity. Immunol Lett 2005;99:24-9

Lan Q, Zhang L, Li G, Vermeulen R, Weinberg RS, Dosemeci M, Rappaport SM, Shen M, Alter BP, Wu Y, Kopp W, Waidyanatha S, Rabkin C, Guo W, Chanock S, Hayes RB, Linet M, Kim S, Yin S, Rothman N, Smith MT. Hematotoxicity in workers exposed to low levels of benzene. Science 2004;306:1774-6

Lan Q, Zhang L, Shen M, Smith MT, Li G, Vermeulen R, Rappaport SM, Forrest MS, Hayes RB, Linet M, Dosemeci M, Alter BP, Weinberg RS, Yin S, Yeager M, Welch R, Waidyanatha S, Kim S, Chanock S, Rothman N. Polymorphisms in cytokine and cellular adhesion molecule genes and susceptibility to hematotoxicity among workers exposed to benzene. Cancer Res 2005;65:9574-81

Lee JY, Kim JY, Lee YG, Shin WC, Chun T, Rhee MH, Cho JY. Hydroquinone, a reactive metabolite of benzene, reduces macrophage-mediated immune responses. Mol Cells 2007;23:198-206

Rothman N, Smith MT, Hayes RB, Traver RD, Hoener B, Campleman S, Li GL, Dosemeci M, Linet M, Zhang L, Xi L, Wacholder S, Lu W, Meyer KB, Titenko-Holland N, Stewart JT, Yin S, Ross D. Benzene poisoning, a risk factor for hematological malignancy, is associated with the NQO1 609C-->T mutation and rapid fractional excretion of chlorzoxazone. Cancer Res 1997;57:2839-42

Wacholder S, Chanock S, Garcia-Closas M, El GL, Rothman $\mathrm{N}$. Assessing the probability that a positive report is false: an approach for molecular epidemiology studies. J Natl Cancer Inst 2004;96:434-42

Yoon BI, Li GX, Kitada K, Kawasaki Y, Igarashi K, Kodama Y, Inoue T, Kobayashi K, Kanno J, Kim DY, Inoue T, Hirabayashi Y. Mechanisms of benzene-induced hematotoxicity and leukemogenicity: cDNA microarray analyses using mouse bone marrow tissue. Environ Health Perspect 2003;111:1411-20

Zeger SL, Liang KY. Longitudinal data analysis for discrete and continuous outcomes. Biometrics 1986;42:121-30

Zhang L, Tinkle SS. Chemical activation of innate and specific immunity in contact dermatitis. J Invest Dermatol 2000;115:168-76 